\title{
MATCHED PAIRS OF LIE ALGEBROIDS
}

\author{
by TAHAR MOKRI
}

(Received 16 October, 1995)

\begin{abstract}
We extend to Lie algebroids the notion variously known as a double Lie algebra (Lu and Weinstein), matched pair of Lie algebras (Majid), or twilled extension of Lie algebras (Kosmann-Schwarzbach and Magri). It is proved that a matched pair of Lie groupoids induces a matched pair of Lie algebroids. Conversely, we show that under certain conditions a matched pair of Lie algebroids integrates to a matched pair of Lie groupoids. The importance of matched pairs of Lie algebroids has been recently demonstrated by Lu.
\end{abstract}

Introduction. The notion of a Lie bialgebra, due to Drinfel'd [5], has arisen in the study of Poisson Lie groups. A Poisson Lie group $G$, that is a Lie group $G$ with a compatible Poisson bracket \{\} , induces a Lie algebra structure on the vector space dual $\mathscr{G}^{*}$ of the Lie algebra $\mathscr{G}$ of $G$, such that the direct sum $\mathscr{G} \oplus \mathscr{G}^{*}$ of vector spaces acquires a Lie algebra structure with $\mathscr{G}$ and $\mathscr{G}^{*}$ as Lie subalgebras. If $G^{*}$ is the connected and simply connected Lie group whose Lie algebra is $\mathscr{G}^{*}$, then it is sometimes possible to equip the product manifold $G \times G^{*}$ with a Lie group structure whose Lie algebra is $\mathscr{G} \oplus \mathscr{G}^{*}$, in such a way that the triple $\left(G, G^{*}, G \times G^{*}\right)$ becomes a matched pair of Lie groups [17]. Abstracting the structures of $G \times G^{*}$ and $\mathscr{G} \oplus \mathscr{G}^{*}$ leads to the concepts of matched pairs of Lie groups and matched pairs of Lie algebras.

A matched pair of Lie groups is a triple $(V, H, G)$ of three Lie groups $V, H$ and $G$ such that $V$ and $H$ are Lie subgroups of $G$, and the map $V \times H \rightarrow G$ defined by $(v, h) \mapsto v h$ is a diffeomorphism. Similarly a double Lie algebra is a triple $(\mathscr{V}, \mathscr{H}, \mathscr{G})$ of three Lie algebras $\mathscr{V}, \mathscr{H}$ and $\mathscr{G}$ such that $\mathscr{G}=\mathscr{V} \oplus \mathscr{H}$ as vector spaces, and $\mathscr{V}, \mathscr{H}$ are Lie subalgebras of $\mathscr{G}$. Matched pairs of Lie groups and matched pairs of Lie algebras are also natural extensions of semi direct products of Lie groups and of their Lie algebras; this is the case where one action is trivial.

Matched pairs of Lie groups and matched pairs of Lie algebras have arisen in the work of several authors in connection with Poisson geometry and dressing transformations, notably Kosmann-Schwarzbach and Magri in [7], where matched pairs of Lie algebras are studied under the name of twilled extensions of Lie algebras, Majid in [17] (see also [18]), and Lu and Weinstein in [11]. In [12] Lu and Weinstein showed that the Lie bialgebra $\left(\mathscr{G}, \mathscr{G}^{*}\right)$ associated with any Poisson Lie group $G$ may be integrated to a double Lie groupoid in the sense of [14].

Mackenzie [14] extended the concept of double Lie group to the context of Lie groupoids, showing that there is a double Lie groupoid structure associated with a double Lie group (this is not as obvious as the terminology makes it seem). It has been also shown [14] that the double groupoids which arise from double Lie groups can be characterized amongst general double groupoids by a simple condition called vacancy. Since the concept of a double Lie groupoid is more general than that of a vacant double groupoid the terminology matched pairs of Lie groupoids is more appropriate for the groupoid version of a double Lie group. Although the paper [14] studied the infinitesimal

Glasgow Math. J. 39 (1997) 167-181. 
actions associated with a matched pair of Lie groupoids, it did not consider any notion of matched pair for Lie algebroids.

In this paper we introduce a natural notion of matched pair of Lie algebroids and show that a matched pair of Lie groupoids induces a matched pair of Lie algebroids. This process is significantly more involved than for matched pairs of Lie groups and algebras. The Lie algebroid representations which constitute the matched pair may be obtained either by linearizing the groupoid actions and then applying the Lie functor, or by linearizing the infinitesimal actions on the Lie algebroid level. The importance of matched pairs of Lie groupoids and Lie algebroids has recently been dramatically reaffirmed by new work of $\mathrm{Lu}[10]$, who shows that matched pairs underlie any Poisson action of a Poisson Lie group.

The article is organized as follows: in Section 1 we review matched pairs of Lie groupoids. In Sections 2 and 3 we study the induced algebroid actions of $A V$ on $H$ and $A H$ on $V$, where $(V, H)$ is a matched pair of Lie groupoids. These actions yield representations of the Lie algebroids $A V$ and $A H$ on each other by "twisted derivations". In Section 4 we define a matched pair of abstract Lie algebroids by a Lie algebroid structure on the vector bundle direct sum, exactly as for double Lie algebras. In Section 5 we prove that the Lie algebroids $A V$ and $A H$ of a matched pair $V$ and $H$ of Lie groupoids form a matched pair of Lie algebroids. As an example, we show that for a compact Poisson Lie group $G$, the Lie algebroid $T^{*} G$ of 1 forms on $G$, and the Lie algebroid $T G$ associated to the tangent bundle $T G \rightarrow G$ form a matched pair of Lie algebroids. In Section 6 we prove an integration result for matched pairs of Lie algebroids, modelled on a result by Lu and Weinstein ([11], Section 3.7). Some further integration results will be given in another paper.

All manifolds are $C^{\infty}$, real, Hausdorff, and second countable. A Lie groupoid $\Omega$ over base $B$ with the source map $\alpha: \Omega \rightarrow V$, and the target map $\beta: \Omega \rightarrow B$ is denoted by $\Omega \stackrel{\alpha, \beta}{\rightrightarrows} B$. The identity map of $\Omega$ is denoted by $1^{\Omega}$ and its value at $b \in B$ by $1_{b}^{\Omega}$, or simply by $1_{b}$. The notations $V$ and $H$ are always for two Lie groupoids with the same base $B$; we denote by $v_{1}, v_{2}, \ldots, v_{n}, \ldots$, the elements of $V$ and by $h_{1}, h_{2}, \ldots, h_{n}, \ldots$ the elements of $H$. We use the maps $\alpha_{V}, \beta_{V}, \alpha_{H}$ and $\beta_{H}$ for the source and the target maps of $V$ and $H$ respectively, but we omit the subscripts $V$ and $H$ if there is no confusion in doing so. Finally, we denote by $C(M)$ the module of smooth real valued maps on a manifold $M$.

1. Matched pairs of Lie groupoids. In this section, we review briefly matched pairs of Lie groupoids. More details may be found in [14] (see also [19]).

Definition 1.1. [14]. Let $V$ and $H$ be two Lie groupoids over the same base $B$. The groupoids $V$ and $H$ form a matched pair of Lie groupoids, or $(V, H)$ is a matched pair of Lie groupoids, if there exits a left action $(h, v) \mapsto{ }^{h} v$ of $H$ on $V$ and a right action $(h, v) \mapsto h^{v}$ of $V$ on $H$ such that the following conditions are satisfied

(i) $\alpha_{V}\left({ }^{h} v\right)=\beta_{H}\left(h^{v}\right)$ for all $(h, v)$ with $\alpha_{H}(h)=\beta_{V}(v)$;

(ii) ${ }^{h}\left(v_{1} v_{2}\right)=\left({ }^{h} v_{1}\right)\left({ }^{h{ }^{v}} v_{2}\right)$ with $\alpha_{V}\left(v_{1}\right)=\beta_{V}\left(v_{2}\right)$ and $\alpha_{H}(h)=\beta_{V}\left(v_{1}\right)$;

(iii) $\left(h_{1} h_{2}\right)^{v}=\left(h_{1}^{h_{2 v}}\right)$. $\left(h_{2}^{v}\right)$ for $v \in V, h_{1}, h_{2} \in H$ with $\alpha_{H}\left(h_{1}\right)=\beta_{H}\left(h_{2}\right)$.

The following two properties are a consequence of the properties (ii) and (iii) in the above definition: 
(iv) ${ }^{h}\left(1_{b}^{V}\right)=1_{c}^{V}$, for $h \in H$ with $\alpha_{H}(h)=b$ and $\beta_{H}(h)=c$;

(v) $\left(1_{c}^{H}\right)^{v}=1_{b}^{H}$, for $v \in V$ with $\beta_{V}(v)=c$ and $\alpha_{V}(v)=b$.

For two Lie groupoids $V$ and $H$ with the same base $B$, we denote by $V * H$ the submanifold $\left\{(v, h) \in V \times H \mid \alpha_{V}(v)=\beta_{H}(h)\right\}$ of $V \times H$. If $V$ and $H$ form a matched pair of Lie groupoids, then we denote by $\Phi:(h, v) \mapsto \Phi_{h}(v)$ and by $\Psi:(h, v) \mapsto \Psi_{v}(h)$ the left action of $H$ on $V$ and the right action of $V$ on $H$, respectively.

Proposition 1.2 [14] (p. 199, Theorem 2.10). $(V, H)$ is a matched pair of Lie groupoids if and only if the manfold $G=V * H$ has a Lie groupoid structure on base $B$, such that

(i) the maps $v \mapsto\left(v, 1_{\alpha(v)}^{H}\right)$ and $h \mapsto\left(1_{\beta(h)}^{V}, h\right)$ are Lie groupoid morphisms from $V$ and $H$ to $G$, respectively. We denote by $V^{\prime}$ and by $H^{\prime}$ the images of $V$ and $H$ under such morphisms.

(ii) The multiplication $m: V^{\prime} * H^{\prime} \rightarrow g$ is a diffeomorphism, where $V^{\prime} * H^{\prime}$ is the set of all composable pairs $\left(v^{\prime}, h^{\prime}\right) \in V^{\prime} \times H^{\prime}$.

We recall [13] that if a Lie group $G$ acts on a manifold $M$, one can construct a Lie groupoid structure on the product manifold $G \times M$ with base $M$, called the action groupoid. This structure is described as follows: $\alpha$ is the projection onto the second factor of $G \times M$ and $\beta$ is the action $G \times M \rightarrow M$ itself; the identity map is $m \mapsto(1, m)$; the inverse of $(g, m)$ is $\left(g^{-1}, g m\right)$ and the partial multiplication is $(g, m)\left(g^{\prime}, m^{\prime}\right)=\left(g g^{\prime}, m^{\prime}\right)$, for $m=g^{\prime} m^{\prime}$. We recall also that for any manifold $B$, there is a Lie groupoid structure on the product manifold $B \times B$, with base $B$, called the coarse groupoid structure. The source and the target maps are the projections of $B \times B$ onto the second and the first factor, respectively, and the multiplication is defined by $\left(b_{1}, b_{2}\right)\left(b_{3}, b_{4}\right)=\left(b_{1}, b_{4}\right)$, for $b_{2}=b_{3}$. Now the next proposition gives a class of examples of a matched pair of Lie groupoids; see [19] for others.

Proposition 1.3. Let $H$ be a Lie group, and $\Phi: H \times M \rightarrow M,(h, m) \mapsto h m$, an action of $H$ on a manifold $M$. Then the action groupoid $H \ltimes M$, and the coarse groupoid $M \times M$ form a matched pair of Lie groupoids over base $M$.

Proof. If $\tilde{H}=H \ltimes M$ and $\tilde{V}=M \times M$, the pullback

$$
\tilde{H} * \tilde{V}=\left\{(\tilde{h}, \tilde{v}) \in \tilde{H} \times \tilde{V} \mid \alpha_{\tilde{H}}(\tilde{h})=\beta_{\tilde{v}}(\tilde{v})\right\}
$$

is just the trivial Lie groupoid $M \times H \times M$. The maps $(h, m) \mapsto(h m, h, m)$ and $(x, y) \mapsto$ $(x, 1, y)$ are groupoid morphisms, and represent $\tilde{H}$ and $\tilde{V}$ embedded as wide subgroupoids in $M \times H \times M$. The multiplication of an element of $\tilde{H}$ by an element of $\tilde{V}$ is

$$
(h m, h, m)(m, 1, x)=(h m, h, x) .
$$

For $(y, h, m) \in M \times H \times M$ we have $\left(y, h, h^{-1} y\right)\left(h^{-1} y, 1, x\right)=(y, h, x)$, in a unique way, with $\left(y, h, h^{-1} y\right) \in \tilde{H}$, and $\left(h^{-1} y, 1, x\right) \in \tilde{V}$. It follows from the Proposition 1.2 , that $(\tilde{H}, \tilde{V})$ is a matched pair of Lie groupoids.

If $G$ is a Lie groupoid on base $B$ with $m$ as multiplication, the tangent bundle $T G$ equipped with $T m$ as multiplication is then a Lie groupoid over $T B$. Assume now that 
$(V, H)$ is a matched pair of Lie groupoids, and let $m$ be the multiplication in $V * H$. Since $T(V * H)=T V * T H$, there is a Lie groupoid structure on $T V * T H$, such that the maps: $X \mapsto(X, T(1) T \alpha X)$ and $Y \mapsto(T(1) T \beta Y, Y)$ represent $T V$ and $T H$ as embedded wide subgroupoids in $T(V * H)$. The proof of the following proposition follows now from the Proposition 1.2.

Proposition 1.4. If $(V, H)$ is a matched pair of Lie groupoids then $(T V, T H)$ is also a matched pair of Lie groupoids.

2. Lie algebroids. Here we briefly recall the concept of Lie algebroid. $A$ Lie algebroid on base $B$ is a vector bundle $q: A \rightarrow B$ together with a map $a: A \rightarrow T B$ of vector bundles over $B$, called the anchor of $A$, and an $\mathbb{R}$-bilinear, antisymmetric bracket of sections, [, ]: $\boldsymbol{A} \times \Gamma A \rightarrow \Gamma A$, which obey the Jacobi identity, and satisfies the relations (i) $a[X, Y]=[a(X), a(Y)]$ and (ii) $[X, f Y]=f[X, Y]+(a(X) f) Y)$, for $X, Y \in \Gamma A, f \in$ $C(B)$. Here $a(X)(f)$ is the Lie derivative of $f$ with respect to the vector field $a(X)$.

Let $A$ be a Lie algebroid on $B$ and let $f: M \mapsto B$ be a smooth map. Then an action of $A$ on $M$ is an $\mathbb{R}$-linear map $X \mapsto X^{\dagger}, \Gamma A \rightarrow \Gamma A$ such that (i) $[X, Y]^{\dagger}=\left[X^{\dagger}, Y^{\dagger}\right]$, for $X, Y \in \Gamma A$; (ii) $(u X)^{\dagger}=(u \circ f) X^{\dagger}$ for $X \in \Gamma A, u \in C(B)$; (iii) $T f\left(X^{\dagger}(m)\right)=a(X)(f)(m)$ for $X \in \Gamma A, m \in M$.

The construction of the Lie algebroid of a Lie groupoid follows closely the construction of a Lie algebra of a Lie group; we refer to [13] for full account. Let $G \rightrightarrows B$ be a Lie groupoid, and let $T^{\alpha} B=\operatorname{Ker}(T \alpha)$ be the vertical bundle along the fibers of $\alpha$. Let $A G \rightarrow B$ be the vector bundle pullback of the vector bundle $T^{\alpha} G$ across the identity map $1: B \rightarrow G$. Notice that a section $X \in \Gamma A G$ is characterized by $X(b) \in T_{1_{b}} G_{b}, \forall b \in B$, where $G_{b}=\alpha^{-1}(b)$. Now take $X \in \Gamma A G$ and denote by $\vec{X}$ the right invariant vector field on $G$, defined by $\vec{X}(g)=T R_{g} X(\beta(g))$; the correspondence $X \mapsto \vec{X}$ from $\Gamma A G$ to the module of right invariant vector fields is a bijection; we equip $\Gamma A G$ with the Lie algebra structure obtained by transferring the Lie algebra structure of the module of right invariant vector fields on $G$ to $\Gamma A G$, via the bijection $X \mapsto \vec{X}$. Namely, if $X, Y \in \Gamma A G$, we define $[X, Y]=[\vec{X}, \vec{Y}] \circ 1$, and $a: A G \rightarrow T B$, by $a\left(X_{b}\right)=T \beta X_{b}$. The vector bundle $A G$ constructed above is called the Lie algebroid of $G$; we will denote it by $q_{G}: A G \rightarrow B$, and its anchor map by $a_{G}: A G \rightarrow T B$. If there is no confusion we will omit the subscript. The pullback of $\vec{X}$ by the inversion map $i$ of $G$, is denoted by $\bar{X}$ and is a left invariant vector field on $G$. Lastly, if $\left(x_{t}\right)$ is the one parameter group of local diffeomorphisms which generates a right invariant vector field $\vec{X}$ on $G$, then $x_{t}(v)=x_{t}\left(1_{\beta(v)}\right) v, \forall v \in G$, and we write $x_{t}(v)=\exp t X(\beta(v)) v$, where $\exp t X(b)=x_{t}\left(1_{b}\right)$.

Now assume that $(V, H)$ is a matched pair of Lie groupoids. The left action $\Phi$ of $H$ on $\beta: V \rightarrow B$ induces an action of the Lie algebroid $A H$ on the groupoid $V$. This action associates to $Y \in \Gamma A H$ the vector field $Y^{\dagger}$ on $V$, called the fundamental vector field generated by $Y$, defined by:

$$
Y^{\dagger}(v)=T\left(h \mapsto \Phi_{h}(v)\right) Y(\beta(v)), \forall v \in V,
$$

and a left action of $H$ on $q: A V \rightarrow B$, defined by:

$$
\chi_{h}(X)=T i T \Phi_{h}(T i X)
$$

for all $(h, X) \in H \times A V$ with $\alpha_{H}(h)=q_{V}(X)$.

Similarly, the right action $\Psi$ of $V$ on $\alpha: H \rightarrow B$, induces an infinitesimal action of the 
Lie algebroid $A V$ on $H$. This action associates to $X \in \Gamma A V$ the vector field $X^{\dagger}$ on $H$ defined by:

$$
X^{\dagger}(h)=T\left(v \mapsto \Psi_{v}(h)\right) T i X(\alpha(h)), \forall h \in H,
$$

and a right action of the Lie groupoid $V$ on $q: A H \rightarrow B$, given by the formula:

$$
\chi_{v}^{\prime}(Y)=T \Psi_{v} Y,
$$

for all $(Y, v) \in A H \times V$ such that $q_{H}(Y)=\beta_{V}(v)$. Finally, we will denote by $m$ and $m^{\prime}$ the multiplications in the groupoids $V$ and $H$ respectively.

Lastly, we have

Proposition 2.1 [14] (p. 224, Theorem 4.9). If $(V, H)$ is a matched pair of Lie groupoids, then for $Y \in \Gamma A H, X \in \Gamma A V, v$ and $w$ in $V, h$ and $l$ in $H$, such that $\alpha(v)=\beta(w)$ and $\alpha(h)=\beta(l)$, the following relations are satisfied:

$$
Y^{\dagger}(v w)=\operatorname{Tm}\left(Y^{\dagger}(v),\left(\chi_{v}^{\prime}(Y(\beta(v)))^{\dagger}(w)\right)\right.
$$

and

$$
X^{\dagger}(h l)=T m^{\prime}\left(\left(\chi_{l}(X(\alpha(l)))^{\dagger}(h), X^{\dagger}(l)\right) .\right.
$$

Notice that from 1.1 the vector fields $Y^{\dagger}$ and $X^{\dagger}$ satisfy the relations

$$
Y^{\dagger}\left(1_{b}^{H}\right)=T\left(1^{H}\right) a_{H}(Y)(b)
$$

and

$$
X^{\dagger}\left(1_{b}^{v}\right)=T\left(1^{v}\right) a_{V}(X)(b)
$$

3. The representations induced by a matched pair. For a matched pair $(V, H)$ of Lie groupoids, there are induced representations of the Lie algebroids $A V$ and $A H$ on each other by "twisted observations", exactly as in the Lie algebra case. We recall now from [6] the definition of a representation of a Lie algebroid on a vector bundle. Let $A$ be a Lie algebroid over $B$ with anchor map $a: \mathscr{A} \rightarrow T B$. Let $E$ be a vector bundle over $B . A$ representation of $\mathscr{A}$ on $E$ is a $\mathbb{R}$ bilinear map

$$
\rho: \Gamma \mathscr{A} \times \Gamma E \rightarrow \Gamma E:(X, \mu) \mapsto \rho_{X}(\mu),
$$

such that for all $X, Y \in \Gamma \mathscr{A}, \mu \in \Gamma E$ and $f \in C(B)$,

(i) $\rho_{f X}(\mu)=f \rho_{X}(\mu)$;

(ii) $\rho_{X}(f \mu)=f \rho_{X}(\mu)+(a(X) f) \mu$;

(iii) $\rho_{[X, Y]}(\mu)=\rho_{X}\left(\rho_{Y}(\mu)\right)-\rho_{Y}\left(\rho_{X}(\mu)\right)$.

For a matched pair of Lie groupoids $(V, H)$ and $Y \in \Gamma A H$, let $\rho_{Y}: \Gamma A V \rightarrow \Gamma A V$ be the map defined by:

$$
\rho_{Y}(X)(b)=\left[i, Y^{\dagger}, \vec{X}\right]\left(1_{b}\right), \quad \forall b \in B .
$$

Since $Y^{\dagger}$ is $\beta$ projectable, $i_{*} Y^{\dagger}$ is $\alpha$ projectable and then $\rho_{Y}(X) \in \Gamma A V$. Similarly, $\rho_{X}^{\prime}$ for $X \in \Gamma A V$ is the map $\Gamma A H \rightarrow \Gamma A H$ defined by:

$$
\rho_{X}^{\prime}(Y)(b)=\left[X^{\dagger}, \vec{Y}\right]\left(1_{b}\right), \quad \forall b \in B .
$$

Since the vector field $X^{\dagger}$ is $\alpha$ projectable the map $\rho_{X}^{\prime}$ takes its value in $\Gamma A H$.

Proposition 3.1. The map $Y \mapsto \rho_{Y}$ is a representation of the Lie algebroid $A H$ on the 
vector bundle $A V$, and the map $X \mapsto \rho_{X}^{\prime}$ is a representation of the Lie algebroid $A V$ on the vector bundle $A H$.

LEMmA 3.2. If $W$ is a vector field on $V$ tangent to the $\alpha$ fibres, that is $T \alpha W(v)=0$, for any $v \in V$, then:

$$
\left[i_{*} Y^{\dagger}, \overrightarrow{W \circ 1}\right]\left(1_{b}\right)=\left[i_{*} Y^{\dagger}, W\right]\left(1_{b}\right),
$$

for any $b \in B$, where $\overrightarrow{W \circ 1}$ is the right invariant vector field on $V$ associated to the section $W \circ 1 \in \Gamma A V$.

Proof of the lemma. The vector field $X=W-\overrightarrow{W \circ 1}$ vanishes identically on $1_{B}$ and the relation $i_{*} Y^{\dagger}\left(1_{b}\right)=T(1) T \beta Y\left(1_{b}\right)$ shows that $i_{*} Y^{\dagger}$ restricts to the base $1_{B}$ as a vector field. It follows that $\left[i_{*} Y^{\dagger}, X\right]$ vanishes identically on $1_{B}$.

Proof of the proposition 3.1. For $f \in C(B)$, we have

$$
\begin{aligned}
\rho_{Y}(f X)(b) & =\left[i_{*} Y^{\dagger}, \overrightarrow{f X}\right]\left(1_{b}\right) \\
& =\left[i_{*} Y^{\dagger},(f \circ \beta) \vec{X}\right]\left(1_{b}\right) \\
& =(f \circ \beta)\left[i_{*} Y^{+}, \vec{X}\right]\left(1_{b}\right)+\left(i_{*} Y^{\dagger}(f \circ \beta)\right)_{b} \vec{X}\left(1_{b}\right) .
\end{aligned}
$$

Since $\beta\left(1_{b}\right)=b$ and $\vec{X}\left(1_{b}\right)=X(b)$, for all $b \in B$, we have

$$
\rho_{Y}(f X)(b)=f\left(\rho_{Y}(X)(b)\right)+\left(i_{*} Y^{\dagger}\left(1_{b}\right)(f \circ \beta)\right) X(b) .
$$

But

$$
i_{*} Y^{\dagger}\left(1_{b}\right)(f \circ \beta)=(d f \circ T \alpha) Y^{\dagger}\left(1_{b}\right)=\left(a_{H}(Y) f\right)(b)
$$

then

It follows that

$$
\begin{aligned}
\rho_{Y}(f(X)(b) & =f \rho_{Y}(X)(b)+d f\left(a_{H}(Y)(b)\right) X(b) \\
& =f \rho_{Y}(X)(b)+a_{H}(Y) f(b) X(b) .
\end{aligned}
$$

$$
\rho_{Y}(f X)=f \rho_{Y}(X)+a_{H}(Y)(f) X .
$$

For $f \in C(B)$, we have $(f Y)^{\dagger}=(f \circ \beta) Y^{\dagger}$, by the very definition of $Y^{\dagger}$, therefore

$$
i_{*}(f Y)^{\dagger}=i_{*}(f \circ \beta) Y^{\dagger}=(f \circ \alpha) i_{*} Y^{\dagger} .
$$

It follows, by noticing that

$$
\vec{X}(f \circ \alpha)=d f \circ T \alpha \vec{X}=0,
$$

that

$$
\rho_{f Y}(X) b=f(b) \rho_{Y}(X)(b)
$$

The assertion

$$
\left[\rho_{Y}, \rho_{Y^{\prime}}\right]=\rho_{\left[Y, Y^{\prime}\right]}, \forall Y, Y^{\prime} \in \Gamma A H
$$


follows from thè Jacobi identity and from the relation:

$$
\left[i_{*} Y^{\dagger},\left[i_{*} Y^{\dagger}, \vec{X}\right]\right]\left(1_{b}\right)=\left[i_{*} Y^{\dagger},\left(\overrightarrow{\rho_{Y^{\prime}}(X)}\right)\right]\left(1_{b}\right), \quad \forall b \in B,
$$

which is a consequence of the Lemma 3.2.

The second part of the proof concerning $\rho_{X}^{\prime}$ is proved in the same way.

The following proposition shows that the representations $\rho$ and $\rho^{\prime}$ may also be obtained by linearizing the actions $\Phi$ and $\Psi$, as for the Lie group case.

Proposition 3.3. For all $Y \in \Gamma A H$, all $X \in \Gamma A V$, and all $b \in B$, we have the relations

(i) $\rho_{X}^{\prime}(Y)(b)=\left.\frac{d}{d t}\right|_{t=0} T \Psi_{\exp t X(b)} Y(\beta \circ \exp t X(b))$,

(ii) $T i \rho_{Y}(X)(b)=\left.\frac{d}{d t}\right|_{t=0} T \Psi_{i \exp t Y(b)} T i X(\beta \circ \exp t Y(b))$.

Proof. We prove the first assertion. The second one can be proved by the same argument. Let $F_{t}(h)=\Psi_{i \exp X(\alpha h)}(h)$ be the (local) flow of $X^{\dagger}$. We have

$$
\left[X^{\dagger}, \vec{Y}\right]\left(1_{b}^{H}\right)=\left.\frac{d}{d t}\right|_{t=0} T F_{t}^{-1} Y(\beta \circ(\exp t X)(b)) ;
$$

but

$$
\begin{aligned}
T F_{t}^{-1} Y(\beta \circ(\exp t X(b))) & =\left.\frac{d}{d s}\right|_{s=0} F_{t}^{-1}(\exp s Y(\beta \circ(\exp t X)(b))) \\
& =\left.\frac{d}{d s}\right|_{s=0} \Psi_{\exp t X(b)}(\exp s Y(\beta \circ \exp t X)(b)) \\
& =T \Psi_{\exp t X(b)}(Y(\beta \circ \exp t X)(b)),
\end{aligned}
$$

by using the relation $\exp t X(b)=i(\exp -t X(\beta \circ \exp t X(b)))$; see ([13], Chap. 2, Sect. 5), and that proves the proposition.

We need now the following lemma.

Lemma 3.4. For all $X \in \Gamma A V$, for all $Y \in \Gamma A H$, and for all $b \in B$, the following properties hold:

(i) $(X+T i X(b)=T(1)(T \alpha(X)+T \beta(X))(b)$;

(ii) $\left[Y^{\dagger}, i_{*} \vec{X}\right]\left(1_{b}\right)=-\left[Y^{\dagger}, \vec{X}\right]\left(1_{b}\right)+T(1)\left[a_{H}(Y), a_{V}(X)\right]$;

(iii) $\rho_{Y}(X) b=\left[Y^{\dagger}, \vec{X}\right]\left(1_{b}\right)-T(1) T \alpha\left[Y^{\dagger}, \vec{X}\right]\left(1_{b}\right)$,

(iv) $\rho_{X}^{\prime}(Y) b=\left[i_{*} Y^{\dagger}, \vec{Y}\right]\left(1_{b}\right)-T(1) T \alpha\left[i_{*} Y^{\dagger}, \vec{Y}\right]\left(1_{b}\right)$.

Proof. (i) We have $\operatorname{Tm}(X(b), \operatorname{Ti} X(b))=T(1) T \beta X(b)$, since $T V$ is a Lie groupoid with base $T B$ and multiplication $T m$. But

$$
\begin{aligned}
\operatorname{Tm}(X(b), \operatorname{TiX}(b)) & =\operatorname{Tm}(X(b)-T(1) T \alpha X(b), 0)+\operatorname{Tm}(T(1) \operatorname{T\alpha } X(b), \operatorname{Ti} X(b)) \\
& =X(b)-T(1) \operatorname{T\alpha } X(b)+\operatorname{TiX}(b),
\end{aligned}
$$

thus the result. 
(ii) Let $F_{t}(v)=\Phi_{\exp Y(\beta(v))}(v)$ be the (local) flow of $Y^{\dagger}$, then

$$
\begin{aligned}
{\left[Y^{\dagger}, i_{*} \vec{X}\right]\left(1_{b}\right) } & =\left.\frac{d}{d t}\right|_{t=0} T F_{t}^{-1} T i \vec{X}\left(1_{\beta(\exp t Y(b))}\right) \\
& =\left.\frac{d}{d t}\right|_{t=0}-T F_{t}^{-1} \vec{X}\left(1_{\beta(\exp t Y(b))}\right)+\left.\frac{d}{d t}\right|_{t=0} T F_{t}^{-1} T\left(1^{v}\right) T \beta \vec{X}\left(1_{\beta \circ(\exp t Y(b)))}\right) \\
& =-\left[Y^{+}, \vec{X}\right]\left(1_{b}\right)+T\left(1^{v}\right)\left[a_{H}(Y), a_{V}(X)\right](b),
\end{aligned}
$$

by using the formula $T i X+X=T\left(1^{v}\right) A(X)$, from the first part of the lemma. imply

(iii) Notice that the two relations $T \beta Y^{\dagger}(v)=a_{H}(Y)(\beta v)$ and $T \beta \vec{X}(v)=a_{V} X(\beta v)$

We have

$$
T \beta\left[Y^{\dagger}, \vec{X}\right]\left(1_{b}\right)=\left[a_{H}(Y), a_{V}(X)\right](b), \quad \forall b \in B .
$$

$$
\begin{aligned}
\rho_{Y}(X)(b)= & {\left[i_{*} Y^{\dagger}, \vec{X}\right]\left(1_{b}\right) } \\
= & T i\left[Y^{\dagger}, i_{*} \vec{X}\right]\left(1_{b}\right) \\
= & -T i\left[Y^{\dagger}, \vec{X}\right]\left(1_{b}\right)+T\left(1^{v}\right)\left[a_{H}(Y), a_{V}(X)\right](b)(\text { by }(1)), \\
= & {\left[y^{\dagger}, \vec{X}\right]\left(1_{b}\right)-T\left(1^{v}\right)\left(T \alpha\left[Y^{\dagger}, \vec{X}\right]\left(1_{b}\right)+T \beta\left[Y^{\dagger}, \vec{X}\right]\right)\left(1_{b}\right) } \\
& +T\left(1^{v}\right)\left[a_{H}(Y), a_{V}(X)\right](b)(\text { by }(1) \text { of } 3.4), \\
= & {\left[Y^{\dagger}, \vec{X}\right]\left(1_{b}\right)-T(1) T \alpha\left[Y^{\dagger}, \vec{X}\right]\left(1_{b}\right) . }
\end{aligned}
$$

(iv) The statement for $\rho_{X}^{\prime}(Y)$ is proved by the same method.

Proposition 3.5. If $(V, H)$ is a matched pair of Lie groupoids then the representations $\rho$ and $\rho$ ' satisfy the following relations:

$$
\overrightarrow{\rho_{Y}(X)}(v)=\left[Y^{\dagger}, \vec{X}\right](v)+\left(\rho_{X}^{\prime}(Y)\right)^{\dagger}(v)
$$

for all $v$ in $V$, and

$$
\overrightarrow{\rho_{X}^{\prime}(Y)}(h)=\left[i_{*} X^{\dagger}, \vec{Y}\right](h)+i_{*}\left(\rho_{Y}(X)\right)^{\dagger}(h)
$$

for all $h \in H$.

Proof. Let $\vec{X}$ be a right invariant vector field on $V$ with $X \in \Gamma A V$, and let $f_{t}$ be its (local) flow. We recall that

$$
f_{t}(v w)=f_{t}(v) w, \quad \forall u, w \in V, \text { such that } \alpha(v)=\beta(w),
$$

with $f_{t}(v)=\exp t X(\beta(v)) v$; it follows that if $Z$ and $W$ are two vector fields in $V$, with $T \alpha Z_{v}=T \beta W_{w}$, then the following relation holds

$$
T f_{t} \operatorname{Tm}\left(Z_{v}, W_{w}\right)=\operatorname{Tm}\left(T f_{t} Z_{v}, W_{w}\right) .
$$

Now if in the relation (1) we replace $v$ by $f_{t}\left(1_{b}\right)$, with $b=\beta(w)$, and then if we apply this operator $T f_{t}^{-1}$ to both sides of this relation by $T f_{t}^{-1}$, we get

$$
\left(f_{t}\right)^{*} Y^{\dagger}(w)=T m\left(\left(f_{t}\right)^{*} Y^{\dagger}\left(1_{b}\right), \quad T\left(h \rightarrow \Phi_{h}(v)\right) T \Psi_{f_{t}\left(1_{b}\right)} Y\left(\beta f_{t}\left(1_{b}\right)\right)\right) .
$$


Differentiating the above relation with respect to $t$ at $t=0$, we get

$$
-\left[Y^{\dagger}, \vec{X}\right](w)=\operatorname{Tm}\left(-\left[Y^{\dagger}, \vec{X}\right]\left(1_{b}\right), \rho_{X}^{\prime}(Y)^{\dagger}(w)\right),
$$

by the Proposition 3.3. Now the above relation may be written as follows

$$
\begin{aligned}
{\left[Y^{\dagger}, \vec{X}\right](w)=} & \operatorname{Tm}\left(\left[Y^{\dagger}, \vec{X}\right]\left(1_{b}\right),-\rho_{X}^{\prime}(Y)^{\dagger}(w)\right) \\
= & \operatorname{Tm}\left(\left[Y^{\dagger}, \vec{X}\right]\left(1_{b}\right)-T(1) T \alpha\left[Y^{\dagger}, \vec{X}\right]\left(1_{b}\right), 0\right) \\
& +\operatorname{Tm}\left(T(1) T \alpha\left[Y^{\dagger}, \vec{X}\right]\left(1_{b}\right),-\rho_{X}^{\prime}(Y)^{\dagger}(w)\right) \\
= & \overrightarrow{\rho_{Y}(X)}(w)-\left(\rho_{X}^{\prime}(Y)\right)^{\dagger}(w),
\end{aligned}
$$

by the Lemma 3.4 .

From the relation (2) we deduce similarly

$$
i_{*} X^{\dagger}(h l)=\operatorname{Tm}^{\prime}\left(i_{*} X^{\dagger}(h), T i T\left(v \rightarrow \Psi_{v}(i l) T \Phi_{i h} T i X(\beta h)\right),\right.
$$

and if $\vec{Y}$ is any right invariant vector field on $H$, then we have by the same argument

$$
\begin{aligned}
{\left[i_{*} X^{\dagger}, \vec{Y}\right](h) } & =\operatorname{Tm}^{\prime}\left(\left[i_{*} X^{\dagger}, \vec{Y}\right]\left(1_{a}\right),-i_{*} \rho_{Y}(X)^{\dagger}(h)\right) \\
& =\overrightarrow{\rho_{X}^{\prime}(Y)}(h)-i_{*}\left(\rho_{Y}(X)\right)^{\dagger}(h),
\end{aligned}
$$

where $a=\beta(h)$.

THEOREM 3.6. If $(V, H)$ is a matched pair of Lie groupoids and if $\rho$ and $\rho^{\prime}$ are the induced representations defined by (5) and (6), then for all $Y, W \in \Gamma A H$, and all $X, Z \in \Gamma A V$, the following relations are satisfied

(i) $\rho_{Y}[X, Z]=\left[\rho_{Y}(X), Z\right]+\left[X, \rho_{Y}(Z)\right]-\rho_{\rho_{X}(Y)}(Z)+\rho_{\rho_{Z}^{\prime}(Y)}(X)$,

(ii) $\rho_{X}^{\prime}[Y, W]=\left[\rho_{X}^{\prime}(Y), W\right]+\left[Y, \rho_{X}^{\prime}(W)\right]-\rho_{\rho_{Y}(X)}^{\prime}(W)+\rho_{\rho_{W}(X)}^{\prime}(Y)$.

(iii) $a_{V}\left(\rho_{Y}(X)\right)-a_{H}\left(\rho_{X}^{\prime}(Y)\right)=\left[a_{H}(Y), a_{V}(X)\right]$.

Proof. To avoid overloading notations, we shall denote here by the same letter a section of the Lie algebroid $A V$ and the corresponding invariant vector field on $V$.

(i) and (ii). From the relation (7) we deduce that

(a) $\rho_{Y}[X, Z]=\left[Y^{\dagger},[X, Z]\right]+\left(\rho_{[X, Z]}^{\prime}(Y)\right)^{+}$

(b) $\left[\rho_{Y}(X), Z\right]=\left[\left[Y^{\dagger}, X\right], Z\right]+\left[\left(\rho_{X}^{\prime}(Y)\right)^{\dagger}, Z\right]$;

(c) $\left[X, \rho_{Y}(Z)\right]=\left[X,\left[Y^{\dagger}, Z\right]\right]+\left[X,\left(\rho_{Z}^{\prime}(Y)\right)^{\dagger}\right]$;

(d) $\rho_{\rho_{Z}^{\prime}(Y)}(X)=\left[\left(\rho_{Z}^{\prime}(Y)\right)^{\dagger}, X\right]+\left(\rho_{X}^{\prime}\left(\rho_{Z}^{\prime}(Y)\right)\right)^{\dagger}$

(e) $\rho_{\rho_{X}^{\prime}(Y)}(Z)=\left[\left(\rho_{X}^{\prime}(Y)\right)^{\dagger}, Z\right]+\left(\rho_{Z}^{\prime}\left(\rho_{X}^{\prime}(Y)\right)\right)^{\dagger}$.

If we add the equations (b), (c) and (d) and then subtract (e), we get the equation (a), by using the Jacobi identity and the relation $\rho_{[X, Z]}^{\prime}=\rho_{X}^{\prime} \rho_{Z}^{\prime}-\rho_{Z}^{\prime} \rho_{Z}^{\prime}$. We prove the similar assertion for $\rho$ by the same method.

(iii) The relation (7) gives

$$
\alpha_{\nu} \rho_{Y}(X) b=T \beta\left[Y^{\dagger}, \vec{X}\right]\left(1_{b}\right)+T \beta\left(\rho_{X}^{\prime}(Y)\right)^{\dagger}\left(1^{b}\right) ;
$$

but $T \beta\left[Y^{\dagger}, \vec{X}\right]\left(1_{b}\right)=\left[a_{H}(Y), a_{V}(X)\right](b), \forall b \in B$. As the vector field $Y^{\dagger}$ on $V$ is $\beta$ projectable to the vector field $a_{H}(Y)$ on $B$, and $\left(\rho_{X}^{\prime}(Y)\right)^{\dagger}\left(1_{b}^{V}\right)=T\left(1^{V}\right)\left(a_{H} \rho_{X}^{\prime}(Y)(b)\right)$ by (3), the result follows.

In the next section we will see that the equations proved in the Theorem 3.6 may be used to define an abstract notion of matched pairs of Lie algebroids. 
We will prove later that under some topological assumptions, a matched pair of Lie algebroids is integrable to a matched pair of Lie groupoids, provided that the Lie algebroids arise from Lie groupoids. The last assumption is crucial since not all Lie algebroids are integrable.

4. Matched pairs of Lie algebroids. The following definition is an extension to Lie algebroids of the definition of a double Lie algebra in [11] or a matched pair of Lie algebras in [17], or of a twilled extension of Lie algebras in [7].

Definition 4.1. Two Lie algebroids $\mathscr{A}$ and $\mathscr{B}$, with the same base $B$ form a matched pair of Lie algebroids if the direct sum $\mathscr{C}=\mathscr{A} \oplus \mathscr{B}$, of vector bundles has a Lie algebroid structure on base $B$, such that $\mathscr{A}$ and $\mathscr{B}$ are Lie subalgebroids of $\mathscr{C}$.

The following theorem shows that a matched pair of Lie algebroids $(\mathscr{A}, \mathscr{B})$ induces a representation of $\mathscr{A}$ on $\mathscr{B}$ and a representation of $\mathscr{B}$ on $\mathscr{A}$, and these two representations are by "twisted derivations", as in the Lie algebra case.

THEOREM 4.2. If the Lie algebroids $\mathscr{A}$ and $\mathscr{B}$ form a matched pair of Lie algebroids, there exists a representation

$$
\rho: \Gamma \mathscr{B} \times \Gamma \mathscr{A} \rightarrow \Gamma \mathscr{A},
$$

of $\mathscr{B}$ on $\mathscr{A}$ and a representation

$$
\rho^{\prime}: \Gamma \mathscr{A} \times \Gamma \mathscr{B} \rightarrow \Gamma \mathscr{B}
$$

of $\mathscr{A}$ on $\mathscr{B}$. These two representations satisfy the relations

(i) $\rho_{Y}[X, Z]=\left[\rho_{Y}(X), Z\right]+\left[X, \rho_{Y}(Z)\right]-\rho_{\rho_{\dot{X}}^{\prime}(Y)}(Z)+\rho_{\rho_{\dot{Z}}^{\prime}(Y)}(X)$,

(ii) $\rho_{X}^{\prime}[Y, W]=\left[\rho_{X}^{\prime}(Y), W\right]+\left[Y, \rho_{X}^{\prime}(W)\right]-\rho_{\rho_{Y}(X)}^{\prime}(W)+\rho_{\rho_{W}(X)}^{\prime}(Y)$,

(iii) $a\left(\rho_{Y}(X)\right)-a^{\prime}\left(\rho_{X}^{\prime}(Y)\right)=\left[a^{\prime}(Y), a(X)\right]$.

for all $X, Z \in \Gamma \mathscr{A}$, and $Y, W \in \Gamma \mathscr{B}$.

Proof. Let $\Pi_{1}$ and $\Pi_{2}$ be the projections of $\mathscr{A} \oplus \mathscr{B}$ onto the first and the second factor, respectively. We define $\rho$, and $\rho^{\prime}$ by:

and

$$
\rho_{Y}(X)=-\Pi_{1}[X, Y]
$$

$$
\rho_{X}^{\prime}(Y)=\Pi_{2}[X, Y]
$$

for all $X \in \Gamma \mathscr{A}$ and all $Y \in \Gamma \mathscr{B}$. It follows that:

$$
-\rho_{Y}(X)+\rho_{X}^{\prime}(Y)=[X, Y]
$$

and therefore, the relation (iii) of 4.2 follows by applying the enchor map to the left and right hand side of (9). We have also

and

$$
\rho_{Y}(f X)=f \rho_{Y}(X)+a_{s \mathcal{A}}(X) f Y,
$$

$$
\rho_{f Y}(X)=f \rho_{Y}(X)
$$

for all $f \in C(B)$, by the properties of a Lie bracket. The similar statements for $\rho_{f x}^{\prime}(Y)$ and $\rho_{X}^{\prime}(f Y)$ hold, by the same argument. 
Now for $Y^{\prime} \in \Gamma \mathscr{B}$, we have

$$
\begin{aligned}
-\rho_{\left[Y, Y^{\prime}\right]}(X)+\rho_{X}^{\prime}\left[Y, Y^{\prime}\right]= & {[X,[Y, Y]] } \\
= & {\left[[X, Y], Y^{\prime}\right]+\left[Y,\left[X, Y^{\prime}\right]\right] } \\
= & {\left[-\rho_{Y}(X)+\rho_{X}^{\prime}(Y), Y^{\prime}\right]+\left[Y,-\rho_{Y^{\prime}}(X)+\rho_{X}^{\prime}\left(Y^{\prime}\right)\right] } \\
= & -\left[\rho_{Y}(X), Y^{\prime}\right]+\left[\rho_{X}^{\prime}(Y), Y^{\prime}\right]-\left[Y, \rho_{Y^{\prime}}(X)\right]+\left[Y, \rho_{X}^{\prime}\left(Y^{\prime}\right)\right] \\
= & +\rho_{Y^{\prime}} \rho_{Y}(X)-\rho_{\rho_{Y}(X)}^{\prime}\left(Y^{\prime}\right)+\left[\rho_{X}^{\prime}(Y), Y^{\prime}\right]+\left[Y, \rho_{X}^{\prime}\left(Y^{\prime}\right)\right] \\
& -\rho_{Y} \rho_{Y^{\prime}}(X)+\rho_{\rho_{Y^{\prime}}(X)}^{\prime}(Y)
\end{aligned}
$$

the second equality is by using the Jacobi identity. The relation (ii) of 4.2 and the relation

$$
\left[\rho_{Y}, \rho_{Y^{\prime}}\right]=\rho_{Y} \rho_{Y^{\prime}}-\rho_{Y^{\prime}} \rho_{Y}
$$

follow from the last equation. The similar results for $\rho^{\prime}$ are proved by the same method.

The next theorem is a converse of the Theorem 4.2.

THEOREM 4.3. Let $\mathscr{A}$ and $\mathscr{B}$ be two Lie algebroids with the same base $B$, with anchor maps $a$ and $a^{\prime}$, respectively. If there exist representations $\rho$ and $\rho^{\prime}$ of $\mathscr{B}$ and $\mathscr{A}$ on each other, such that the relations (i) and (ii) and (iii) in the Theorem 4.2 hold, then there is a unique matched pair structure on $\mathscr{A}$ and $\mathscr{B}$ which induces the representations $\rho$ and $\rho$ '.

Proof. Let $a: \mathscr{A} \oplus \mathscr{B} \rightarrow T B$ be defined by $a(X \oplus Y)=a(X)+a^{\prime}(Y)$. We define on $\mathscr{A} \oplus \mathscr{B}$ bracket by setting

$$
[X \oplus 0,0 \oplus Y]=[X, Y]=-\rho_{Y}(X)+\rho_{X}^{\prime}(Y) .
$$

We have then

$$
\begin{aligned}
{\left[X+Y, X^{\prime}+Y^{\prime}\right]=} & {\left[X, X^{\prime}\right]+\rho_{Y}\left(X^{\prime}\right)-\rho_{Y^{\prime}}(X) } \\
& +\left[Y, Y^{\prime}\right]+\rho_{X}^{\prime}\left(Y^{\prime}\right)-\rho_{X^{\prime}}^{\prime}(Y) .
\end{aligned}
$$

Equipped with the above brackets and with the map $a$ is an enchor map the vector bundle $\mathscr{A} \oplus \mathscr{B}$ is a Lie algebroid; the verification is straightforward.

Since the injections of $\mathscr{A}$ and $\mathscr{B}$ in $\mathscr{A} \oplus \mathscr{B}$ are Lie algebroid morphisms $(\mathscr{A}, \mathscr{B})$ is a matched pair of Lie algebroids with $\rho$ and $\rho^{\prime}$ as induced representations. The uniqueness of the Lie algebroid structure on $\mathscr{A} \oplus \mathscr{B}$ with $\rho$ and $\rho^{\prime}$ as induced representations follows from the relation (10).

For a matched pair $(\mathscr{A}, \mathscr{B})$ of Lie algebroids, we denote by $\mathscr{A} \bowtie \mathscr{B}$ the Lie algebroid $\mathscr{A} \oplus \mathscr{B}$, following the convention in use for the Lie algebra case [[17], [11]].

5. Matched pairs of Lie algebroids induced by matched pairs of Lie groupoids. Matched pairs of Lie algebroids arise from matched pairs of Lie groupoids in the same way that matched pairs of Lie algebras arise from matched pairs of Lie groups, by applying the Lie functor to the Lie groupoids. 
In this section $V$ and $H$ are always two Lie groupoids over the same base $B$, and the manifold $G$ is the pullback $V * H$, defined in Section 1. The maps $\alpha_{G}$ and $\beta_{G}$ are defined by $\alpha_{G}(v, h)=\alpha_{H}(h)$, and $\beta_{G}(v, h)=\beta_{V}(v)$. If we denote by $K$ the submanifold $\left\{\left(X_{1_{b}^{v}}, Y_{1_{b}^{\prime \prime}}\right) \in T_{\left(1_{b}^{v}, 1_{b}^{\prime \prime}\right)} G \mid T \alpha_{H} Y_{1_{h}^{\prime \prime}}=0\right\}$ of $T(V \times H)$, then $K$ may be regarded as a vector bundle on $B$ and the module $\Gamma K$ of sections of $K$ may be identified with the submodule

$$
\left\{\left(X+T\left(1^{v}\right) T \beta Y, Y\right) \mid X \in \Gamma A B, Y \in \Gamma A H\right\}
$$

of $\Gamma_{\mid B} T(V \times H)$.

Proposition 5.1. The Lie algebroids $A V$ and $A H$ form a matched pair of Lie algebroids if and only if $K$ has a Lie algebroid structure with base $B$, such that the maps $X \mapsto(X, 0)$, and $Y \mapsto\left(T\left(1^{V}\right) T \beta Y, Y\right)$ are Lie algebroid morphisms, from $A V$ and $A H$ respectively, to $K$.

Proof. Let $F$ be the map $A V \oplus A H \rightarrow K$, defined by

$$
F(X \oplus Y)=\left(X+T\left(1^{V}\right) T \beta Y, Y\right), \forall X \oplus Y \in A V \oplus A H,
$$

then $F$ is clearly a vector bundle isomorphism. This isomorphism carries the Lie algebroid structure from $A V \oplus A H$ to $K$, and vice versa.

Assume that $(A V, A H)$ is a matched pair of Lie algebroids, then $K$ has a Lie algebroid structure isomorphic to $A V \oplus A H$, by the isomorphism $F$. Since the maps $i_{V}: X \mapsto(X, 0)$ and $i_{H}: Y \mapsto(0, Y)$ are Lie algebroid morphisms from $A V$ and $A H$ to $A V \oplus A H$, the maps $i_{V} \circ F$ and $i_{H} \circ F$ are Lie algebroid morphisms, as well.

Conversely, assume that $K$ has a Lie algebroid structure such that $j_{V}: X \mapsto(X, 0)$ and $\left.j_{H}: Y \mapsto T\left(1^{V}\right) T \beta Y, Y\right)$ are Lie algebroid morphisms. We have only to prove that $A V$ and $A H$ are Lie subalgebroids of $A V \oplus A H$; this follows from the relations $i_{V}=F^{-1} \circ j_{V}$, and $i_{H}=F^{-1} \circ j_{H}$.

THEOREM 5.2. If $(V, H)$ is a matched pair of Lie groupoids, then $(A V, A H)$ is a matched pair of Lie algebroids, and for $X \in \Gamma A V, Y \in \Gamma A H$, the bracket $[X, Y]$ is given by

$$
[X, Y]=-\rho_{Y}(X)+\rho_{X}^{\prime}(Y),
$$

where the maps $\rho$ and $\rho^{\prime}$ are the representations defined by the relations (5) and (6).

Proof. The theorem is a consequence of the Theorems 3.6 and 4.3.

Example 5.3. Let $(V, H)$ be a matched pair of Lie groupoids over the base $B$. Assume $H$ is the trivial Lie groupoid $B \times B \rightrightarrows B$, and $V$ is a symplectic groupoid [2]. Then,

$$
A V \oplus A H=T^{*} B \oplus T B,
$$

and $\left(T^{*} B, T B\right)$ is a matched pair of Lie algebroids.

Example 5.4. If $(V, H)$ is a matched pair of Lie groupoids, then $(T A V, T A H)$ is a matched pair of Lie algebroids. Indeed, $(T V, T H)$ is a matched pair of Lie groupoids 
(over $T B$ ), by 1.4 and the Lie algebroids $A T V$ and $A T H$ are isomorphic to the Lie algebroids $T A V$ and $T A H$ by ([15], Theorem 7.1).

ExAmple 5.5. Let $G \times M \rightarrow M$ be an action of a Lie group $G$ on a manifold $M$ and let $\mathscr{G}$ be the Lie algebra of $G$. The trivial vector bundle $\mathscr{G} \times M$ on $M$ has a Lie algebroid structure on base $M$, denoted by $\mathscr{G} \ltimes M$ and called the action Lie algebroid. Furthermore $\mathscr{G} \times M=A(G \ltimes M)[6]$, and now from the Proposition 1.3 the Lie algebroids $T M$ and $\mathscr{G} \times M$ form a matched pair of Lie algebroids.

Let $(P, \pi)$ be a Poisson manifold with Poisson bivector field $\pi$. Let $\pi^{\#}$ be the associated bundle map $\pi^{\sharp}(p): T_{p}^{*} P \rightarrow T_{p} P$ defined by

$$
\left(\alpha_{p}, \pi^{\#}\left(\beta_{p}\right)\right)=\pi(p)\left(\alpha_{p}, \beta_{p}\right) .
$$

Then, with $-\pi^{\#}$ as the anchor map, the cotangent bundle $T^{*} P$ has a Lie algebroid structure over $P$, where the Lie bracket on the space $\Omega^{1}(P)$ of 1 -forms on $P$ is given by

$$
\{\alpha, \beta\}=-D(\pi(\alpha, \beta))-L_{\pi^{*} \alpha} \beta+L_{\pi^{*} \beta} \alpha,
$$

where $L_{\pi^{*} \alpha} \beta$ denotes the Lie derivative of the 1 -form $\beta$ in the direction of the vector field $\pi^{\sharp} \alpha$, see [11] (and the references given there).

For a compact and simply connected Poisson Lie group $G$, we prove that the Lie algebroid $T G$ which arise from the tangent bundle $T G \rightarrow G$, and the Lie algebroid $T^{*} G$ of one forms on $G$, form a matched pair of Lie algebroids.

THEOREM 5.6. Let $G$ be a compact and simply connected Poisson Lie group, with Poisson tensor $\pi$, then $\left(T G, T^{*} G\right)$ is a matched pair of Lie algebroids.

Proof. Let $G^{*}$ be the dual Poisson Lie group of $G$, and let $\mathscr{G}$ and $\mathcal{G}^{*}$ be the Lie algebras of $G$, and $G^{*}$, respectively [5] (see also [11]).

For $\alpha \in G^{*}$, let $\alpha_{l}^{\dagger}=\pi\left(\alpha_{l}\right)$, where $\alpha_{l}$ is the left invariant one form on $G$ with $\alpha_{l}(e)=\alpha$. Since $G$ is compact, the left infinitesimal dressing action of $\mathscr{G}^{*}$ on $G, \alpha \mapsto \alpha_{l}^{\dagger}$ is integrated to a global left action of $G^{*}$ on $G$. If we identify $T^{*} G$ with $G \times \mathscr{G}^{*}$, via right translations $\theta_{g} \in T_{g}^{*} G \mapsto\left(g, \theta_{g} \circ T R_{g}\right)$, then the Lie algebroid structure on $T^{*} G$, defined by $\pi$ coincides with the action Lie algebroid $A\left(G^{*} \bowtie G\right)$, see [9]. It follows from the Proposition 1.3, that $\left(T^{*} G, A(G \times G)\right)$ is a matched pair of Lie algebroids; since $A(G \times G)=T G$, the theorem is now proved.

6. Integration of matched pairs of Lie algebroids. In the theorem of this section we prove that under some topological assumptions, a weakly integrable matched pair of Lie algebroids gives rise to a matched pair of Lie groupoids, generalizing the Theorem 3.7 in [11] (see also [17] for an integration result of a different type).

Definition 6.1. A matched pair of Lie algebroids $(\mathscr{A}, \mathscr{B})$ is weakly integrable if there exist three Lie groupoids $V, H$ and $G$, with the same base $B$, such that $G$ is connected and simply connected, and $\mathscr{A}=A V, \mathscr{B}=A H$, and $A V \oplus A H=A G$.

Let $M$ and $B$ be two manifolds and $\pi: M \rightarrow B$ a surjective submersion. We call $M$ a $\pi$-(simply) connected manifold if for all $b \in B$ the fibers $\pi^{-1}(b)$ are (simply) connected subspaces of $M$.

THEOREM 6.2. Let $(\mathscr{A}, \mathscr{B})$ be a weakly integrable matched pair of Lie algebroids, on 
base $B$. Let $V, H$ and $G$ be the Lie groupoids with Lie algebroids $\mathscr{A}, \mathscr{B}$ and $\mathscr{A} \bowtie \mathscr{B}$, respectively. Then, if $V$ and $H$ are compact, $\alpha$-connected and simply connected, $(V, H)$ is a matched pair of Lie algebroids.

LEMMA 6.3. Under the hypothesis of 6.2 the manifold

$$
V * H=\left\{(v, h) \in V \times H \mid \alpha_{V}(v)=\beta_{H}(h)\right\}
$$

is connected.

Proof. The manifold $B$ is connected, since $B$ is the base of the connected and simply connected Lie groupoid $G$ whose Lie algebroid is $A V \oplus A H$. Let $C=\left\{\left(1_{b}^{V}, 1_{b}^{H}\right) \mid b \in B\right\}$, and $C_{b}=\alpha_{V}^{-1}(b) \times \beta_{H}^{-1}(b)$. Then,

$$
V * H=C \cap\left(\bigcup_{b \in b} C_{b}\right)
$$

Since $C$ and $C_{b}$ are connected for all $b \in B$, and since $C \cap C_{b}$ is not empty, $V * H$ is connected.

Proof of Theorem 6.2. Let $G$ be the connected and simply connected Lie groupoids on base $B$ with Lie algebroid $A V \oplus A H$. Since the $\alpha$ fibers of $V$ and $H$ are simply connected, the Lie algebroid morphisms $X \mapsto(X, 0)$ and $Y \mapsto(0, Y)$ from $A V$ and $A H$ to $A G$ can be integrated to get $V$ and $H$ as wide subgroupoids of $G$ by [16]. Let $m$ be the map $V * H \mapsto G$ defined by $m(v, h)=v h$ and let $\alpha$ and $\beta$ be respectively the source and the target maps of the Lie groupoid $G$. Let $\left(X_{v}, Y_{h}\right) \in T_{(v, h)}(V * H)$ be such that

$$
\operatorname{Tm}\left(X_{v}, Y_{h}\right)=0 .
$$

By taking each side of the relation (11) by $T \alpha$ and then by $T \beta$ we find $T \alpha Y_{h}=T \beta X_{v}=0$. Since the vector tangents $X_{v}$ and $Y_{h}$ are tangent to the $\beta$ fibers and the $\alpha$ fibers respectively, the tangent vectors $T L_{v}^{-1} X_{v}$ and $T R_{h}^{-1} Y_{h}$ are defined and are in the Lie algebroids $A V$ and $A H$, respectively. If we take now simultaneously each side of the relation (11) by $T L_{v}^{-1}$ and by $T R_{h}^{-1}$, we obtain

$$
T L_{v}^{-1} X_{v}+T R_{h}^{-1} Y_{h}=0 .
$$

Since the sum of $A V \oplus A H$ is direct we have

$$
T L_{v}^{-1} X_{v}=T R_{h}^{-1} Y_{h}=0 .
$$

It follows that $X_{v}=Y_{b}=0$, and the map $m$ is an immersion. Since $A V \oplus A H=A G$, we have $\operatorname{dim} G=\operatorname{dim}(V * H)$, hence $m$ is a local diffeomorphism. Since $V * H$ is compact, $m$ is a proper map, and hence a covering map. By the Lemma 6.3, $V * H$ is connected; now $m$ is a covering map from the connected space $V * H$ to the simply connected space $G$, hence $m$ is a diffeomorphism.

ACKNOWLEDGEMENTs. This work was part of my Sheffield University PhD thesis. I would like to thank Dr K. Mackenzie for his help and encouragement in writing it. I have benefited greatly from several conversations with him during my stay at the University of Sheffield. I would like also to thank Professor Y. Kosmann-Schwarzbach for her many 
helpful comments. I acknowledge with gratitude a grant from the Algerian Ministry of Education and from the British Council.

\section{REFERENCES}

1. C. Albert and P. Dazord, Théorie des groupoïdes de Lie, Publication du Département de Mathématiques de l'Université de Lyon (1989), 53-105.

2. A. Coste, P. Dazord and A. Weinstein, Groupoïdes symplectiques, Publications du Département de Mathématiques de l'Université de Lyon 1, 2/A (1987). $113-134$

3. R. Brown, From groups to groupoids: a brief suvery, Bull. London Math. Soc. 19 (1987),

4. J. Dieudonne, Treatise on analysis, Volume 3 (Academic Press, 1972).

5. V. G. Drinfel'd, Hamilton structures on Lie groups, Lie bialgebras and the geometric meaning of the classical Yang-Baxter equations, Soviet Math. Dokl. 27(1) (1983) 68-71.

6. P. J. Higgins and K. Mackenzie, Algebraic constructions in the category of Lie algebroids, J. Algebra 129 (1990), 194-230.

7. Y. Kosmann-Schwarzbach and F. Magri, Poisson Lie groups and complete integrabilitity, I, Ann. Inst. H. Poincaré 49 (1988), 433-460.

8. A. Kumpera and D. C. Spencer, Lie equations, volume 1: General theory, (appendix) (Princeton University Press, 1972).

9. J. H. Lu, PhD thesis, University of California, Berkeley (1990). (1995).

10. J. H. Lu, Lie algebroids associated to Poisson actions, preprint, University of Arizona

11. J. H. Lu and A. Weinstein, Poisson Lie groups, dressing transformations and Bruhat decomposition, J. Differential Geom. 31 (1990) 501-526.

12. J. H. Lu and A. Weinstein, Groupoïdes symplectiques doubles des groupoïdes de Lie Poisson, CR Acad Sci Paris sér. 1, Maths 309 (1989), 951-954.

13. K. Mackenzie, Lie groupoids and Lie algebroids in differential geometry, London Mathematical Society Lecture Note Series, Vol. 124 (Cambridge Univ. Press, Cambridge, 1988).

14. K. Mackenzie, Double Lie algebroids and second order geometry, 1, Advances in Mathematics, 94, No. 2 (1992) 180-239.

15. K. Mackenzie and P. Xu, Lie bialgebroids and Poisson groupoids, Duke Math. J., 73(2) (1994), 415-452. (1995).

16. K. Mackenzie and P. Xu, Integration of Lie bialgebroids, preprint, University of Sheffield

17. S. H. Majid, Matched pairs of Lie groups associated to solutions of the Yang Baxter equations, Pacific J. Math. 141 No 2 (1990), 311-332.

18. S. H. Majid, Physics for algebraists: non-commutative and non-commutative Hopf algebras by a bicrossproduct construction, J. Algebra, 130 (1990), 17-64.

19. T. Mokri, PhD thesis (University of Sheffield, 1995).

School of Mathematics and Statistics,

UNIVERSITY OF SHEFFIELD

SHEFFIELD, S3 7RH, ENGLAND.

EMAIL: t.mokri@sheffield.ac.nk 